clinicians in recognising dying, in particular how to distinguish dying from reversible acute deterioration.

Objectives To critically appraise the literature on biological factors that may be used as prognostic indicators in advanced cancer patients.

Methods A systematically structured review was conducted using three electronic databases. Studies reporting prognostic biomarkers in cancer patients with a median survival of $\leq 90$ days.

Results 30 articles were included. Seven prognostic biological factors demonstrated Grade A evidence (lymphocyte count, white blood cell count, serum C-reactive protein, albumin, sodium, urea and alkaline phosphatase). An additional eleven prognostic factors were identified with Grade B evidence (platelet count, international normalised ratio, serum vitamin B12, prealbumin, bilirubin, cholesterol, aspartate aminotransferase, alanine transaminase, 40 lactate dehydrogenase, pseudocholinesterase and urate). A number of biomarkers were specifically identified in the last two weeks of life but limitations exist.

Conclusions The biology of dying is an important area for future research, with the evidence focused on signs, symptoms and prognostic factors. This review identifies a number of common themes shared amongst advanced cancer patients and highlights candidate biomarkers which may be indicative of a common biological process to dying.

\section{P-114 THE ROLE OF THE INFECTION PREVENTION NURSE IN THE HOSPICE SETTING}

Tammy Bale. LOROS Hospice, Leicester, UK

\subsection{6/bmispcare-2017-hospice.139}

Introduction The role is often carried out by Infection Prevention Link staff who also have the role of providing care to the patients. LOROS changed this profile three years ago by employing a dedicated qualified infection prevention specialist. As most of the patients within the hospice are vulnerable to infection this role was to look at areas which could be improved and reduce the risk of hospice acquired infections and promote patient, staff and visitors' safety.

Aims The aims were to ensure that LOROS was compliant with national and local guidelines on Infection Prevention. To identify any areas for concern or non-compliance and how it could improve the patient's journey and ensure infection prevention and safety were everyone's business.

Method A review of national and local infection prevention literature was undertaken and the Infection Prevention Lead was part of the Infection Prevention Society (IPS) whose vision is that "no person should be harmed by a preventable infection'. They have also produced a suite of infection prevention audits that can be used in all healthcare settings. These were used as a starting point for starting to identify any good/excellent areas of compliance and any areas which may need improvement.

The hospice also wanted to ensure compliance with the Department of Health's Health and Social Care Act 2008 The Hygiene Code. This Act established the CQC and sets out the overall framework for the regulation of health and adult social care activity. Outcome 8 of the CQC was the driving force for some of the changes that were implemented.

Conclusion From the initial audits areas of good practice were identified, for example the general cleanliness of building, staff adherence to uniform/dress code and hand hygiene but they also identified area for improvement. The list below is an example of some of the changes implemented but it is not an exhaustive list and work continues to identify good practice and areas for improvement

The purchase of all new commodes

Fabric riser recliner chairs started to be phased out

Green labels were introduced identifying equipment had been cleaned

Detergent cleaning wipes

Link Staff had a dedicated two day training day

Oak Ward refurbishment

Safer needles

Introduction of Sepsis Pathway

Patient leaflets

Cleaning schedules and policy

Development of an audit Programme.

\section{P-115 TO FEED, OR NOT TO FEED, THAT IS THE QUESTION: MULTI - PROFESSIONAL TEAM TRAINING ON FOOD AND DRINK FOR THE DYING PATIENT}

${ }^{1}$ Clare Finnegan, ${ }^{2}$ Cathy Godfrey, ${ }^{1,2}$ Karen Groves. 'Lancashire Care Foundation Trust, Preston, UK; ${ }^{2}$ Queenscourt Hospice, Southport, UK

10.1136/bmjspcare-2017-hospice. 140

Aim Whether or not someone can, or should, eat and drink in the last hours and days of life is a dilemma faced by patients, their families and staff. A perception that food or drink has been withheld, and consequently contributed to death, can cause significant distress to bereaved relatives. This project aims to equip Health Care Professionals (HCPs) with the skills to assess the wishes and needs of dying patients, and develop a plan of care with patients and their 'families' regarding eating and drinking.

Design Following review of local audits of care of the dying and initial training sessions with palliative care staff, a halfday interactive training session was developed focusing on:

- Clinical assessment and benefits

- Professional guidelines and the law

- Ethical decision making in clinical practice

- Conversations and documentation.

The session was refined following feedback from participants, a teaching resource pack developed and team-teaching utilised to ensure consistent delivery by different facilitators. Training was offered to all HCPs working across a locality in the North of England.

Results Training sessions were fully booked with 323 trained in the first six months. Participants were multi professional (nurse 38\%, health care assistant 25\%, doctor 24\%, other $13 \%$ ) and from a variety of settings (care home 43\%, hospital $32 \%$, community $11 \%$, hospice $14 \%$ ). Feedback was excellent and comments demonstrated the session had been thought provoking and enjoyable.

Lessons Learnt Participants report clinical decision making and the accompanying conversations about eating and drinking for dying patients is challenging. Differentiating between 'food and drink' and 'clinically assisted hydration and nutrition' is valuable.

Uniting staff with different experiences stimulated excellent reflective debate and shared learning. Staff report increased confidence to develop and review plans in accordance with 